\section{Total Sleep Time}

Helga Peter

Marburg, Deutschland

\section{Synonyme}

TST; Gesamtschlafzeit

\section{Definition}

Messtechnischer Begriff; Bezeichnung für die Gesamtschlafzeit, bestehend aus der Summe der im NREM-Schlaf sowie im REM-Schlaf verbrachten Zeit während der polysomnographischen Untersuchung der Hauptschlafphase.

Siehe auch

- \,Polysomnographie und Hypnogramm“

- $>$ „Aktigraphie“ 